Journal of Nonlinear Mathematical Physics

ISSN (Online): 1402-9251 ISSN (Print): 1776-0852

Journal Home Page: https://www.atlantis-press.com/journals/jnmp

\title{
Lie symmetry analysis, conservation laws and analytical solutions of a time-fractional generalized $\mathrm{KdV}$-type equation
}

Xiu-Bin Wang, Shou-Fu Tian, Chun-Yan Qin, Tian-Tian Zhang

To cite this article: Xiu-Bin Wang, Shou-Fu Tian, Chun-Yan Qin, Tian-Tian Zhang (2017) Lie symmetry analysis, conservation laws and analytical solutions of a time-fractional generalized KdV-type equation, Journal of Nonlinear Mathematical Physics 24:4, 516530, DOI: https://doi.org/10.1080/14029251.2017.1375688

To link to this article: https://doi.org/10.1080/14029251.2017.1375688

Published online: 04 January 2021 


\title{
Lie symmetry analysis, conservation laws and analytical solutions of a time-fractional generalized KdV-type equation *
}

\author{
Xiu-Bin Wang ${ }^{\S, \dagger}$, Shou-Fu Tian ${ }^{\S, *, \S, \llbracket, \star}$, Chun-Yan Qin ${ }^{\S}$ and Tian-Tian Zhang ${ }^{*, \S, \sharp}$ \\ ${ }^{\bullet}$ School of Safety Engineering, China University of Mining and Technology, \\ Xuzhou 221116, People's Republic of China \\ $\S$ School of Mathematics and Institute of Mathematical Physics, China University of Mining and Technology, \\ Xuzhou 221116, People's Republic of China \\ II Department of Applied Mathematics and Theoretical Physics, University of Cambridge, Cambridge CB3 OWA, United \\ Kingdom \\ ^sftian@cumt.edu.cn and shoufu2006@126.com (S.F.Tian) \\ *ttzhang@cumt.edu.cn and zhangtiantian_1001@126.com(T.T. Zhang) \\ †wangxiubin123@cumt.edu.cn (X. B. Wang)
}

Received 12 December 2016

Accepted 25 May 2017

\begin{abstract}
Under investigation in this work is the time-fractional generalized $\mathrm{KdV}$-type equation, which occurs in different contexts in mathematical physics. Lie group analysis method is presented to explicitly study its vector fields and symmetry reductions. Furthermore, two straightforward methods are employed to consider its travelling wave solutions and power series solutions, respectively. Finally, based on the new conservation theorem, conservation laws of the equation are well constructed with a detailed derivation.
\end{abstract}

Keywords: The the time-fractional generalized KdV-type equation; Lie group analysis method; Conservation laws; Analytical solution; Travelling wave solutions.

2000 Mathematics Subject Classification: 35Q51, 35Q53, 35C99, 68W30, 74J35.

\section{Introduction}

It is well known that finding an exact solution of a nonlinear partial differential equation is always one of the central themes arising in physics, mathematics and in many scientific fields. In the past few years, much research have been done on the basic of the group theory and its applications to differential equations $[3,15,24,25]$. In recent years, the fractional calculus was widely applied to describe many complex nonlinear phenomena arising in the areas of heat transfer, diffusion, solid mechanics, wave propagation and other topics. Therefore, the fractional differential equations (FDEs) play a more and more important role in describing physics, engineering, and other scientific fields [5, 17, 26, 27] etc. In 2009, Gazizov and Kasatkin [8] extended Lie symmetry approach to investigate several FDEs. The way for some FDEs are also performed with the aid of RiemannLiouville derivative $[6,9,13,20,28,39,40]$.

The celebrated Noether theorem [23] establishes a connection between symmetries and conservation laws of differential equations. Over the past few years, the application of the Noether theorem

\footnotetext{
*Project supported by the Fundamental Research Funds for the Central Universities under Grant No. 2014 XT02.

${ }^{*}$ Corresponding authors.
} 
has been extended by plenty of mathematicians. Ibragimov [16] and Lukashchuk [21] had made a great contribution to find conservation laws for FDEs that does not admit a fractional Lagrangian. Additionally, conservation laws of some FDEs have been presented with the use of the fractional generalization of the Noether operators [10].

In this paper, we would like to consider the following time-fractional generalized KdV-type equation

$$
D_{t}^{\alpha} u+a u_{n x}+b u^{m} u_{x}=0, \quad(0<\alpha \leq 1),
$$

where $u=u(x, t), a, b, m$ are all real parameters, $n$ is a positive integer and $D_{t}^{\alpha} u$ is the RiemannLiouville fractional derivative of order $\alpha$ with respect to the variable $t$. Invariant analysis of the special form for Eq.(1.1) has been investigated. However, to the best of authors' knowledge, Lie symmetry analysis, conservation laws and analytical solutions of Eq.(1.1) have not been reported in the previous papers.

Eq.(1.1) contains a number of important nonlinear partial differential equations (NLEEs) and FDEs $[1,11,14,41,42]$ as its special case. Next, we will present some special cases.

Case 1: Taking $\alpha=1, a=1, b=6, m=1, n=3$, Eq.(1.1) reduces to the classical KdV equation

$$
u_{t}+6 u u_{x}+u_{x x x}=0
$$

which has been found to describe many physical and engineering phenomena [1].

Case 2: Taking $2 m=1, n=3$, Eq.(1.1) can reduce to the time-fractional Schamel-KdV equation

$$
D_{t}^{\alpha} u+b \sqrt{u} u_{x}+a u_{x x x}=0,
$$

which can describe nonlinear propagation of dust-ion-acoustic (DIA) waves in a one-dimensional and unmagnetized plasma [11].

Case 3: Taking $n=2, a=1$, Eq.(1.1) can be transformed into the following time-fractional Burgers equation

$$
D_{t}^{\alpha} u+b u^{m} u_{x}+u_{x x}=0, \quad(0<\alpha \leq 1) .
$$

Its Lie symmetry analysis and exact solution are succinctly constructed in $[28,41]$.

Case 4: Taking $n=5, a=1$, Eq.(1.1) can be reduced to the time-fractional five-order $\mathrm{KdV}$ equations

$$
D_{t}^{\alpha} u+b u^{m} u_{x}+u_{x x x x x}=0, \quad(0<\alpha \leq 1) .
$$

It have been considered by using the Lie symmetry analysis method in [42].

The primary purpose of the present paper is to investigate the symmetry properties, the travelling wave solutions and power series solutions of Eq.(1.1) by means of three important methods, respectively. Additionally, conservation laws of Eq.(1.1) are also derived by using the Lie point symmetries of the equation.

The rest of this paper is structured as follows. In section 2, a brief review of the main definitions and properties of FDEs are presented to provide a convenient reference. In section 3, the general similarity forms and symmetry reductions of Eq.(1.1) are established. In section 4, two important method are presented to succinctly construct analytical solutions of Eq.(1.1). In section 5, conservation laws of Eq.(1.1) are constructed by using the new conservation theorem. Finally, conclusions and discussion are presented in the last section. 


\section{Preliminaries}

In this section, we briefly recall the main procedure to deal with symmetries for FDEs. Firstly, let's consider the symmetries for a FDE of the form

$$
D_{t}^{\alpha} u(x, t)=F\left(t, u, u_{x}, u_{x x}, \cdots\right), \quad(\alpha>0),
$$

in which $D_{t}^{\alpha} u$ is defined as below. Suppose that $u(x, t)$ is a piecewise continuous function on the $(0, \infty)$ and the integrable on any finite sub-interval of $[0, \infty)$. we can use equality of the following Riemann-Liouville derivative [18]

$$
D_{t}^{\alpha} u=\frac{1}{\Gamma(n-\alpha)} \frac{\partial^{n}}{\partial t^{n}} \int_{0}^{t} \frac{u(x, \tau)}{(t-\tau)^{\alpha+1-n}} d \tau ; n-1<\alpha<n, n \in \mathbb{N} .
$$

Additionally, the modified Riemann-Liouville derivative is given by

$$
D_{x}^{\alpha} f(x)=\frac{1}{\Gamma(n-\alpha)} \frac{\partial^{n}}{\partial t^{n}} \int_{0}^{x} \frac{f(\tau)-f(0)}{(t-\tau)^{\alpha+1-n}} d \tau ; n-1<\alpha<n, n \in \mathbb{N} .
$$

Next, let us consider a one-parameter Lie group of infinitesimal transformation

$$
\begin{aligned}
& t^{*}=t+\epsilon \tau(t, x, u)+o\left(\epsilon^{2}\right), \\
& x^{*}=x+\epsilon \xi(t, x, u)+o\left(\epsilon^{2}\right), \\
& u^{*}=u+\epsilon \eta(t, x, u)+o\left(\epsilon^{2}\right), \\
& \frac{\partial^{\alpha} u^{*}}{\partial t^{* \alpha}}=\frac{\partial^{\alpha} u}{\partial t^{\alpha}}+\epsilon \eta^{\alpha t}(t, x, u)+o\left(\epsilon^{2}\right), \\
& \frac{\partial^{n} u^{*}}{\partial x^{* n}}=\frac{\partial^{n} u}{\partial x^{n}}+\epsilon \eta^{n x}(t, x, u)+o\left(\epsilon^{2}\right), \quad n=1,2,3, \cdots,
\end{aligned}
$$

where $\epsilon$ is a group parameter. The exact expressions of $\eta^{n x}$ are given by

$$
\begin{aligned}
& \eta^{x}=D_{x}(\eta)-u_{t} D_{x}(\tau)-u_{x} D_{x}(\xi), \\
& \eta^{2 x}=D_{x}\left(\eta^{x}\right)-u_{x x} D_{x}(\xi)-u_{x t} D_{x}(\tau), \cdots
\end{aligned}
$$

in which the total derivative operator $D_{x}$ is defined by

$$
D_{x}=\frac{\partial}{\partial x}+u_{x} \frac{\partial}{\partial u}+u_{x x} \frac{\partial}{\partial u_{x}}+\cdots,
$$

with the associated Lie algebra represented by

$$
X=\xi(x, t, u) \frac{\partial}{\partial x}+\tau(x, t, u) \frac{\partial}{\partial t}+\eta(x, t, u) \frac{\partial}{\partial u},
$$

here the coefficient functions $\xi, \tau, \eta$ are to be known later.

If the vector field (2.7) can generate a symmetry of Eq.(1.1), then $X$ have to meet the condition $\left.\operatorname{Pr}^{(n)} X(\Delta)\right|_{\Delta=0}=0$, where $\Delta=D_{t}^{\alpha} u-F\left(t, u, u_{x}, u_{x x}, \cdots\right)$. Let the lower limit of the integral be fixed in (2.2), the explicit form of $\eta^{\alpha t}$ can be explicitly obtained by using Leibnitz rule. Then, by applying prolongation of the fractional vector field to Eq.(1.1), the Lie point symmetries of Eq.(1.1) canbe spanned by the corresponding vector fields. Eq.(1.1) should be invariant with regard to such a transformation (2.4). The invariance condition must arrive at $\left.\tau(t, x, u)\right|_{t=0}=0$. 
According to [8], the developed infinitesimal $\eta^{\alpha t}$ have the following form

$$
\eta^{\alpha t}=D_{t}^{\alpha}(\eta)+\xi D_{t}^{\alpha}\left(u_{x}\right)-D_{t}^{\alpha}\left(\xi u_{x}\right)+D_{t}^{\alpha}\left(D_{t}(\tau) u\right)-D_{t}^{\alpha+1}(\tau u)+D_{t}^{\alpha+1}(u),
$$

in which $D_{t}^{\alpha}$ is the total fractional derivative operator. According to the generalized Leibnitz rule in Ref. [22], we have

$$
D_{t}^{\alpha}(f(t) g(t))=\sum_{n=0}^{\infty}\left(\begin{array}{l}
\alpha \\
n
\end{array}\right) D_{t}^{\alpha} f(t) D_{t}^{n-\alpha} g(t)
$$

where

$$
\left(\begin{array}{l}
\alpha \\
n
\end{array}\right)=\frac{(-1)^{n-1} \Gamma(n-\alpha)}{\Gamma(1-\alpha) \Gamma(n+1)} .
$$

In view of (2.8),(2.9) and (2.10), we have

$$
\eta^{\alpha t}=D_{t}^{\alpha}(\eta)-\alpha D_{t}(\tau) D_{t}^{\alpha} u-\sum_{n=0}^{\infty}\left(\begin{array}{l}
\alpha \\
n
\end{array}\right) D_{t}^{n}(\xi) D_{t}^{\alpha-n}\left(u_{x}\right)-\sum_{n=1}^{\infty}\left(\begin{array}{c}
\alpha \\
n+1
\end{array}\right) D_{t}^{n+1}(\tau) D_{t}^{\alpha-n}(u) .
$$

Linking the generalized chain rule for a composite function [27] of the form

$$
\frac{d^{n} g(f(t))}{d t^{n}}=\sum_{k=0}^{n} \sum_{r=0}^{k}\left(\begin{array}{l}
k \\
r
\end{array}\right) \frac{1}{k !}[-f(t)]^{r} \frac{d^{n}}{d t^{n}}\left[(f(t))^{k-r}\right] \frac{d^{k} g(f(t))}{d f(t)^{k}},
$$

and the Leibnitz rule (2.9), it is easy to obtain the explicit form of $\eta^{\alpha t}$

$$
\begin{aligned}
\eta^{\alpha t} & =\sum_{n=1}^{\infty}\left[\left(\begin{array}{l}
\alpha \\
n
\end{array}\right) \frac{\partial^{n} \eta_{u}}{\partial t^{n}}-\left(\begin{array}{c}
\alpha \\
n+1
\end{array}\right) D_{t}^{n+1}(\tau)\right] D_{t}^{\alpha-n}(u)+\frac{\partial^{\alpha} \eta}{\partial t^{\alpha}}+\left[\eta_{u}-\alpha D_{t}(\tau)\right] \frac{\partial^{\alpha u}}{\partial t^{\alpha}}-u \frac{\partial^{\alpha} \eta_{u}}{\partial t^{\alpha}} \\
& -\sum_{n=1}^{\infty}\left(\begin{array}{l}
\alpha \\
n
\end{array}\right) D_{t}^{n}(\xi) D_{t}^{\alpha-n}\left(u_{x}\right)+\mu .
\end{aligned}
$$

where

$$
\mu=\sum_{n=2}^{\infty} \sum_{m=2}^{n} \sum_{k=2}^{m} \sum_{r=0}^{k-1}\left(\begin{array}{l}
\alpha \\
n
\end{array}\right)\left(\begin{array}{l}
n \\
m
\end{array}\right)\left(\begin{array}{l}
k \\
r
\end{array}\right) \frac{1}{k !} \frac{t^{n-\alpha}}{\Gamma(n+1-\alpha)}(-1)^{r} u^{r} \frac{\partial^{m}}{\partial t^{m}}\left(u^{k-r}\right) \frac{\partial^{n-m-k} \eta}{\partial t^{n-m} \partial u^{k}} .
$$

In the next section, the above detailed analysis of a FDE is applied to investigate the symmetry properties of time-fractional generalized KdV-type equation (1.1).

\section{Symmetry group analysis}

According to the Lie theory, applying the prolongation $\mathrm{pr}^{n}$ to Eq.(1.1), we can obtain the following expression

$$
\eta_{\alpha}^{0}+a \eta^{n x}+b u^{m} \eta^{x}+b m \eta u^{m-1} u_{x}=0 .
$$

Inserting (2.5) and (2.13) into (3.1), then collecting the coefficients of various power of partial derivatives of $u$, we obtain some determining equations. By solving these obtained equations, we 
have

$$
\xi=c_{1} x+c_{2}, \quad \tau=\frac{n c_{1}}{\alpha} t, \quad \eta=\frac{(1-n) c_{1}}{m} u,
$$

where $c_{1}, c_{2}$ are arbitrary constants. According to (3.2), we obtain the corresponding vector fields

$$
X=\left(c_{1} x+c_{2}\right) \frac{\partial}{\partial x}+\frac{n c_{1} t}{\alpha} \frac{\partial}{\partial t}+\frac{(1-n) c_{1} u}{m} \frac{\partial}{\partial u} .
$$

Via the above discussion, the following assertion holds.

Theorem 3.1. The Lie point symmetries of the time-fractional generalized KdV-type equation (1.1) is spanned by the following vector fields

$$
X_{1}=\frac{\partial}{\partial x}, X_{2}=x \frac{\partial}{\partial x}+\frac{n t}{\alpha} \frac{\partial}{\partial t}+\frac{(1-n) u}{m} \frac{\partial}{\partial u} .
$$

According to (3.4), it is not hard to check that the vector fields (3.4) are closed under the Lie bracket, respectively

$$
\left[X_{1}, X_{1}\right]=0, \quad\left[X_{1}, X_{2}\right]=X_{1}, \quad\left[X_{2}, X_{1}\right]=-X_{1}, \quad\left[X_{2}, X_{2}\right]=0 .
$$

For the generator $X_{1}$, we can obtain the invariant solutions $u=a t^{\alpha-1}$ of Eq.(1.1), where $a$ is an arbitrary constant.

For the generator $X_{2}$, we can obtain the characteristic equation

$$
\frac{d x}{x}=\frac{\alpha d t}{n t}=\frac{m d u}{(1-n) u}
$$

and the corresponding invariant

$$
A=x t^{-\frac{\alpha}{n}}, u=t^{\frac{\alpha(1-n)}{n m}} G(A) .
$$

Through the above discussion, we find that Eq.(1.1) can be transformed into a nonlinear fractional ordinary differential equation (FODE). In order to achieve our aim, to begin with, let us introduce the following Erdély-Kober fractional differential operator [19]

$$
\left(p_{\beta}^{\tau, \alpha} G\right)=\prod_{j=0}^{n-1}\left(\tau+j-\frac{\xi}{\beta} \frac{d}{d \xi}\right)\left(K_{\beta}^{\tau+\alpha, n-\alpha} G\right)(A)
$$

where

$$
n= \begin{cases}{[\alpha]+1,} & \alpha \in \mathbb{N}, \\ \alpha, & \alpha \in \mathbb{N},\end{cases}
$$

and

$$
\left(K_{\beta}^{\tau+\alpha, n-\alpha} G\right)(A)=\left\{\begin{array}{l}
\frac{1}{\Gamma(\alpha)} \int_{1}^{\infty}(u-1)^{\alpha-1} u^{-(\tau+\alpha)} G\left(A u^{1 / \beta}\right) d u, \\
G(A), \quad \alpha=0,
\end{array}\right.
$$

is the Erdély-Kober fractional integral operator. 
In view of the definition of the Riemann-Liouville fractional derivative (2.2), we obtain

$$
D_{t}^{\alpha} u=\frac{\partial^{\alpha} u}{\partial t^{\alpha}}=\frac{1}{\Gamma(n-\alpha)} \frac{\partial^{n}}{\partial t^{n}}\left[\int_{0}^{t}(t-s)^{n-\alpha-1} s^{\frac{\alpha(1-n)}{n m}} h\left(x s^{-\frac{\alpha}{2}}\right) d s\right], n-1<\alpha<n, n=1,2,3,4, \ldots
$$

If taking $\rho=\frac{t}{s}$, we get $d s=-t / \rho^{2} d \rho$. Then, Eq.(3.11) can be rewritten as

$$
D_{t}^{\alpha} u=\frac{\partial^{\alpha} u}{\partial t^{\alpha}}=\frac{1}{\Gamma(n-\alpha)} \frac{\partial^{n}}{\partial t^{n}}\left[t^{n+\frac{\alpha(1-n)}{n m}-\alpha} \int_{0}^{t}(\rho-1)^{n-\alpha-1} \rho^{-n-1-\frac{\alpha(1-n)}{n m}+\alpha} h\left(A \rho^{\frac{\alpha}{n}}\right) d s\right] .
$$

By comparing (3.10) with (3.7), we have

$$
\begin{aligned}
& D_{t}^{\alpha} u=\frac{\partial^{\alpha} u}{\partial t^{\alpha}}=\frac{\partial^{n}}{\partial t^{n}}\left[t^{n-\frac{\alpha}{2 m}-\alpha}\left(K_{\frac{n}{\alpha}}^{1+\frac{\alpha(1-n)}{n m}-\alpha, n-\alpha} G\right)(A)\right]=\frac{\partial^{n-1}}{\partial t^{n-1}}\left[\frac{\partial}{\partial t}\left(t^{n+\frac{\alpha(1-n)}{n m}-\alpha}\left(K_{\frac{n}{\alpha}}^{1+\frac{\alpha(1-n)}{n m}, n-\alpha} G\right)\right]\right. \\
& =\ldots=\left(P_{\frac{n}{\alpha}}^{1+\frac{\alpha(1-n)}{n m}-\alpha, \alpha}\right)(A) .
\end{aligned}
$$

Based on the detailed analysis, the following assertion is easily constructed.

Theorem 3.2 Based on the transformation (3.7), Eq.(1.1) can be reduced to a nonlinear FODE

$$
\left(P_{\frac{n}{\alpha}}^{1+\frac{\alpha(1-n)}{n m}-\alpha, \alpha}\right)(A)+a G_{n A}+b G^{m} G_{A}=0
$$

where $n$ is a positive integer and $a, b, m>0$ are arbitrary constants.

Based on Theorem 3.2, Eq.(1.3), Eq.(1.4) and Eq.(1.5) can be reduced to the following FODEs

$$
\begin{aligned}
& \left(P_{\frac{3}{\alpha}}^{1-\frac{7 \alpha}{3}, \alpha}\right)(A)+a G_{3 A}+b G G_{A}=0 \\
& \left(P_{\frac{2}{\alpha}}^{1-\frac{\alpha}{2 m}-\alpha, \alpha}\right)(A)+G_{2 A}+b G^{m} G_{A}=0 \\
& \left(P_{\frac{5}{\alpha}}^{1-\frac{4 \alpha}{m}, \alpha}\right)(A)+G_{5 A}+b G^{m} G_{A}=0
\end{aligned}
$$

respectively.

In particular, if $\alpha=\frac{1}{2}, n=5, m=1$, Eq.(1.1) can be transformed to the following FODE

$$
\left(P_{10}^{\frac{1}{10}, \frac{1}{3}}\right)(A)+a G_{5 A}+b G G_{A}=0
$$

In a similar way, based on Theorem 3.1 and Theorem 3.2, the symmetries and FODEs of Eq.(1.1) can be obtained successively in terms of the specific parameters $\alpha, m, n$.

\section{Analytical solutions}

In this section, two important methods and the algebraic and differential manipulation $[2,4,7,12,29$ $38,43-48]$ are employ to derive the travelling wave solutions and power series solutions of Eq.(1.1). 


\subsection{The power series method}

First of all, let us introduce a important transformation

$$
u(x, t)=u(\epsilon), \quad \epsilon=k x-\frac{\omega t^{\alpha}}{\Gamma(1+\alpha)},
$$

where $k, \omega$ are two arbitrary constants. Substitution of (4.1) into (1.1) leads to the following nonlinear ODE

$$
-\omega u^{\prime}+a k^{n} u_{n \epsilon}+b k u^{m} u^{\prime}=0 .
$$

Then integrating Eq.(4.2) with respect to $\epsilon$ once again, we obtain

$$
-\omega u+a k^{n} u_{(n-1) \epsilon}+\frac{b k}{m+1} u^{m+1}+B=0
$$

where $B$ is an integration constant. let us assume that the solution of Eq.(4.3) has the following form

$$
u=\sum_{s=0}^{\infty} p_{s} \epsilon^{s}
$$

where $p_{s}$ are constants to be known later. Substitution of (4.4) into Eq.(4.3) arrives at the following expression

$$
-\omega \sum_{s=0}^{\infty} p_{s} \epsilon^{s}+a k^{n} \sum_{s=0}^{\infty}(s+1)(s+2) \ldots(s+n-1) p_{s+n-1} \epsilon^{s}+\frac{b k}{m+1}\left(\sum_{s=0}^{\infty} p_{s} \epsilon^{s}\right)^{m+1}+B=0 .
$$

By comparing coefficients of $\epsilon^{s}(s \geq 0)$, we obtain

$$
\begin{aligned}
& p_{n-1}=\frac{1}{a k^{n}(n-1) !}\left(\omega p-\frac{b k}{m+1} p_{0}^{m+1}-B\right), \\
& p_{s+n-1}=\frac{1}{a k^{n}(s+1)(s+2) \ldots(s+n-1)}\left[\omega p_{s}-\frac{b k}{m+1} \sum_{i_{1}+i_{2}+\ldots+i_{m+1}=s} p_{i_{1}} p_{i_{2}} \ldots p_{i_{n-1}}\right] .
\end{aligned}
$$

Thus, any coefficient $p_{s}(s \geq 2)$ of the solution (4.4) are determined by $a, b, p_{0}, \ldots, p_{n-1}, \omega, k$. It shows that there is a power series solution for Eq.(4.2). Additionally, it is not hard to prove the convergence of the power series solution (4.4) with the coefficients depend on (4.6). Obviously, the power series solution (4.4) is an analytical power series solution of Eq.(4.2). Once the power series solution of Eq.(4.2) in hand, the solution of Eq.(1.1) can be easily obtain

$$
\begin{aligned}
u(x, t)= & p_{0}+p_{1}\left(k x-\frac{\omega t^{\alpha}}{\Gamma(\alpha+1)}\right)+\ldots+\frac{1}{a k^{n}(n-1) !}\left(\omega p-\frac{b k}{m+1} p_{0}^{m+1}-B\right)\left(k x-\frac{\omega t^{\alpha}}{\Gamma(\alpha+1)}\right)^{n-1} \\
& +\frac{1}{a k^{n}(s+1)(s+2) \ldots(s+n-1)}\left[\omega p_{s}-\frac{b k}{m+1} \sum_{i_{1}+i_{2}+\ldots+i_{m+1}=s} p_{i_{1}} p_{i_{2}} \ldots p_{i_{n-1}}\right]\left(k x-\frac{\omega t^{\alpha}}{\Gamma(\alpha+1)}\right)^{n+s-1} .
\end{aligned}
$$

Via the above analysis, the following assertion is easily established.

Theorem 4.1 Eq.(1.1) admits the following power series solution

$$
u=\sum_{s=0}^{\infty} p_{s}\left(k x-\frac{\omega t^{\alpha}}{\Gamma(\alpha+1)}\right)^{s}
$$


in which $a, b, p_{0}, \ldots, p_{n-2}, \omega, k$ are some arbitrary constants, the other coefficients $p_{s}(s \geq n-1)$ rely on (4.6).

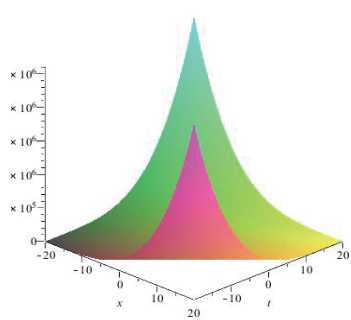

(a)

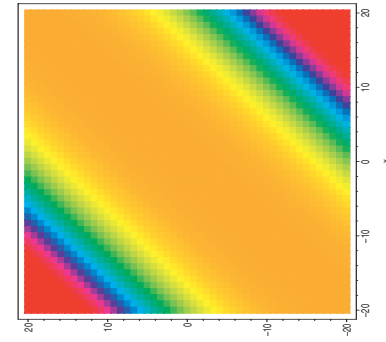

(b)

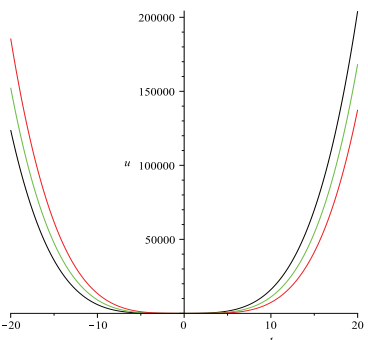

(c)

Figure 1. (Color online) The power series solution (4.8) of Eq.(1.1) by choosing suitable parameters: $k=$ $1, \omega=1, a=1, b=1, n=3, m=4, s=4, \alpha=1$, (a) Perspective view of the real part of the wave. (b) The overhead view of the wave. (c) The wave propagation pattern of the wave along the $t$ axis.

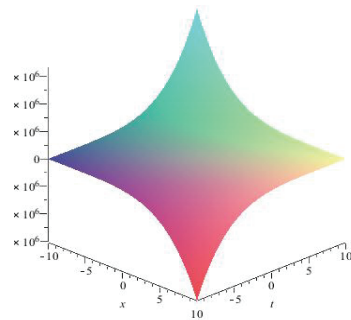

(a)

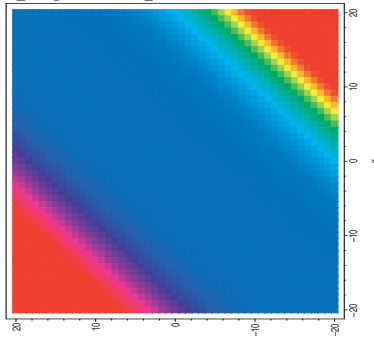

(b)

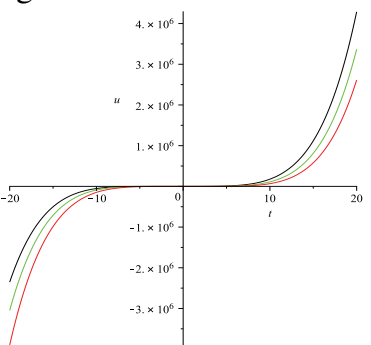

(c)

Figure 2. (Color online) The power series solution (4.8) of Eq.(1.1) by choosing suitable parameters: $k=1, \omega=1, a=1, b=1, n=3, m=4, s=5, \alpha=1$, (a) Perspective view of the real part of the wave. (b) The overhead view of the wave. (c) The wave propagation pattern of the wave along the $t$ axis.

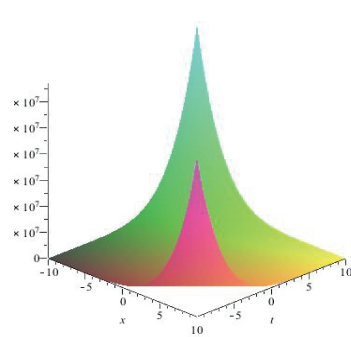

(a)

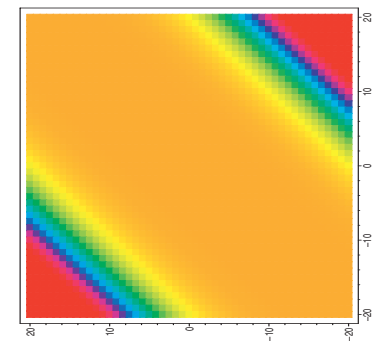

(b)

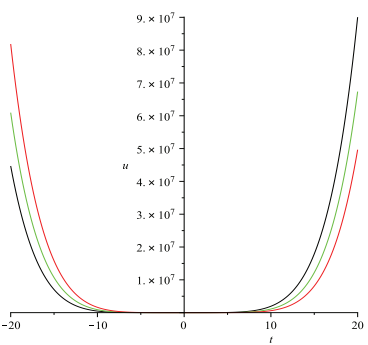

(c)

Figure 3. (Color online) The power series solution (4.8) of Eq.(1.1) by choosing suitable parameters: $k=1, \omega=1, a=1, b=1, n=3, m=4, s=6, \alpha=1$, (a) Perspective view of the real part of the wave. (b) The overhead view of the wave. (c) The wave propagation pattern of the wave along the $t$ axis.

In what follows in order to help us analyse the properties of the analytical solutions well, Figs. 1-3 of the power series solutions (4.8) are plotted by choosing the appropriate parameters. 


\subsection{Fractional sub-equation method}

According to the the fractional sub-equation approach, to begin with, let me introduce the following transformation

$$
u(x, t)=u(\Omega), \Omega=x+c t,
$$

where $c$ is a constant. Substitution of (4.9) into (1.1) leads to the following nonlinear ODE

$$
c^{\alpha} D_{\Omega}^{\alpha} u+a u_{n \Omega}+b u^{m} u_{\Omega}=0 .
$$

Next, by equating the highest order derivative terms and nonlinear terms in (4.10), one has

$$
A+n=A m+A+1 \Leftrightarrow m A=n-1 .
$$

For simplicity, we only consider the following cases $(n \leq 3)$.

Case 1: If $n=2, m=1$, we have $A=1$, based on the fractional sub-equation approach, Eq. (4.2) has the following formal solution

$$
u=c_{0}+c_{1} \phi, \quad c_{1} \neq 0
$$

Inserting Eq.(4.12) along with the Riccati equation $D_{\Omega}^{\alpha} \phi=\sigma+\phi^{2}$ into (4.10), and taking the coefficients of $\phi^{i}(i=0,1,2,3)$ to be zero, we obtain some algebraic equations, then solving the obtained algebraic equations, we get

$$
a=a, b=b, \alpha=\alpha, c=\left(-b c_{0}\right)^{\frac{1}{\alpha}} \quad c_{0}=c_{0}, \sigma=\sigma, c_{1}=-\frac{2 a}{b} .
$$

According to Step 2 in Ref. [48], we obtain the travelling wave solutions of Eq.(1.1) as follow

$$
\begin{aligned}
& u_{1}(x, t)=c_{0}+\frac{2 a}{b} \sqrt{-\sigma} \tanh _{\alpha}(\sqrt{-\sigma} \Omega), \sigma<0, \\
& u_{2}(x, t)=c_{0}+\frac{2 a}{b} \sqrt{-\sigma} \operatorname{coth}_{\alpha}(\sqrt{-\sigma} \Omega), \sigma<0, \\
& u_{3}(x, t)=c_{0}-\frac{2 a}{b} \sqrt{\sigma} \tan _{\alpha}(\sqrt{\sigma} \Omega), \sigma>0, \\
& u_{4}(x, t)=c_{0}+\frac{2 a}{b} \sqrt{\sigma} \cot _{\alpha}(\sqrt{\sigma} \Omega), \sigma>0, \\
& u_{5}(x, t)=c_{0}+\frac{2 a}{b} \frac{\Gamma(1+\alpha)}{\Omega^{\alpha}+\omega}, \omega \text { is a const, } \sigma=0 .
\end{aligned}
$$

where $\Omega=x+c t, a, b, c_{0}, \sigma$ are all arbitrary constants, and the generalized hyperbolic and trigonometric functions are given by

$$
\begin{aligned}
& \tanh _{\alpha}(x)=\frac{\sinh _{\alpha}(x)}{\cosh _{\alpha}(x)}, \operatorname{coth}_{\alpha}(x)=\frac{\cosh _{\alpha}(x)}{\sinh _{\alpha}(x)}, \sinh _{\alpha}(x)=\frac{E_{\alpha}\left(x^{\alpha}\right)-E_{\alpha}\left(-x^{\alpha}\right)}{2}, \\
& \cosh _{\alpha}(x)=\frac{E_{\alpha}\left(x^{\alpha}\right)+E_{\alpha}\left(-x^{\alpha}\right)}{2}, \tan _{\alpha}(x)=\frac{\sin _{\beta}(x)}{\cos _{\beta}(x)}, \cot _{\alpha}(x)=\frac{\cos _{\beta}(x)}{\sin _{\beta}(x)}, \\
& \sin _{\alpha}(x)=\frac{E_{\alpha}\left(i x^{\alpha}\right)-E_{\alpha}\left(-i x^{\alpha}\right)}{2 i}, \cos _{\alpha}(x)=\frac{E_{\alpha}\left(i x^{\alpha}\right)+E_{\alpha}\left(-i x^{\alpha}\right)}{2},
\end{aligned}
$$


and the Mittag-Leffler function is defined by

$$
E_{\alpha}(\alpha)=\sum_{k=0}^{\infty} \frac{z^{k}}{\Gamma(1+k \alpha)} .
$$

Case 2: If $n=3, m=2$, we have $A=1$, thus Eq. (4.2) has the following formal solution

$$
u=c_{0}+c_{1} \phi, \quad c_{1} \neq 0 .
$$

Inserting Eq.(4.17) along with the equation $D_{\Omega}^{\alpha} \phi=\sigma+\phi^{2}$ into (4.17), and collecting the coefficients of $(\phi)^{i}(i=0,1,2,3,4)$ to be zero, Then tackling the obtained algebraic equations, we get

$$
a=a, b=b \quad c_{0}=0, c_{1}^{2}=-\frac{6 a}{b}, c=(-2 a \sigma)^{\frac{1}{\alpha}}, \sigma=\sigma .
$$

In a similar way, the travelling wave solutions of Eq.(1.1) is of the following form

$$
\begin{aligned}
& u_{1}(x, t)=-\sqrt{-\frac{6 a}{b}} \sqrt{-\sigma} \tanh _{\alpha}(\sqrt{-\sigma} \Omega), \sigma<0, \\
& u_{2}(x, t)=-\sqrt{-\frac{6 a}{b}} \sqrt{-\sigma} \operatorname{coth}_{\alpha}(\sqrt{-\sigma} \Omega), \sigma<0, \\
& u_{3}(x, t)=\sqrt{-\frac{6 a}{b}} \sqrt{\sigma} \tan _{\alpha}(\sqrt{\sigma} \Omega), \sigma>0, \\
& u_{4}(x, t)=-\sqrt{-\frac{6 a}{b}} \sqrt{\sigma} \cot _{\alpha}(\sqrt{\sigma} \Omega), \sigma>0, \\
& u_{5}(x, t)=\sqrt{-\frac{6 a}{b}} \frac{\Gamma(1+\alpha)}{\Omega^{\alpha}+\omega}, \omega \text { is a const, } \sigma=0,
\end{aligned}
$$

where $\Omega=x+c t, a, b, c_{0}, \sigma$ are all arbitrary constants, and the generalized hyperbolic and trigonometric functions are defined in Eq.(4.15).

Case 3: If $n=3, m=1$, we have $A=2$, the exact solution of this case has been presented in [39].

In the following, in order to help us analyse the properties of the travelling wave solutions well, Figs.4-5 of the travelling wave solutions ((4.14) and (4.19)) are plotted by choosing the appropriate parameters.

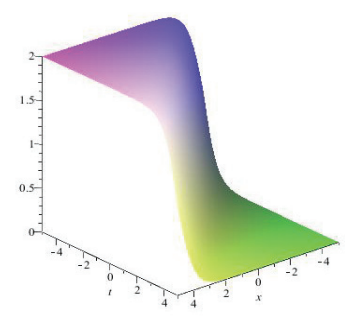

(a)

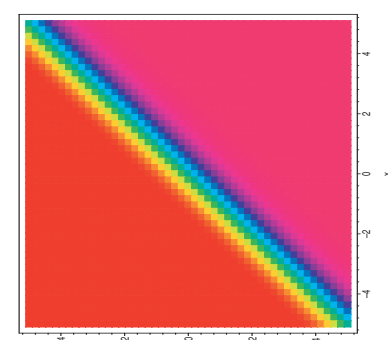

(b)

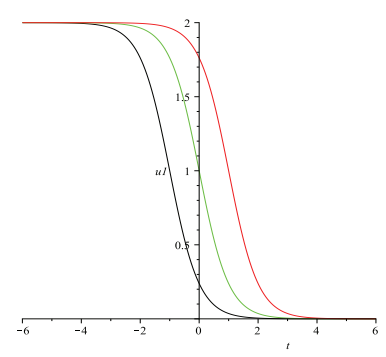

(c)

Figure 4. (Color online) The solution $u_{1}$ (4.14) of Eq.(1.1) by choosing suitable parameters: $c_{0}=1, a=1, b=$ $1, \alpha=1, \sigma=-1$, (a) Perspective view of the real part of the wave. (b) The overhead view of the wave. (c) The 
wave propagation pattern of the wave along the $t$ axis.

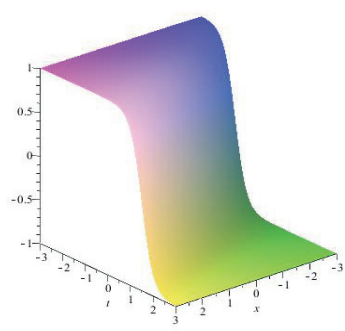

(a)

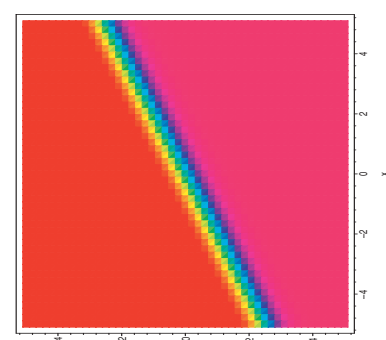

(b)

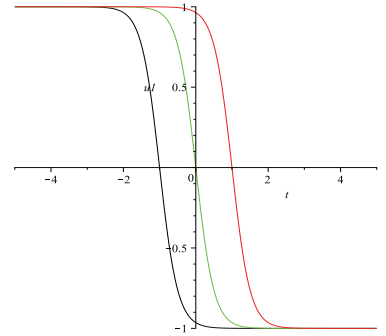

(c)

Figure 5. (Color online) The solution $u_{1}$ (4.19) of Eq.(1.1) by choosing suitable parameters: $c_{0}=0, a=$ $-1, b=6, \sigma=-1, \alpha=1$, (a) Perspective view of the real part of the wave. (b) The overhead view of the wave. (c) The wave propagation pattern of the wave along the $t$ axis.

\section{Conservation laws}

In the present section, the conservation laws of Eq.(1.1) are derived on the basic of the Lie point symmetry (3.4).

Based on the definition of conserved vector for inter-order PDEs, a conserved vector $\mathcal{I}=\left(\mathcal{I}^{t}, \mathcal{I}^{x}\right)$ for Eq.(1.1) admits the following conservation equation

$$
D_{t}\left(\mathcal{I}^{t}\right)+\left.D_{x}\left(\mathcal{I}^{x}\right)\right|_{(1.1)}=0
$$

It is not hard to find that Eq.(1.1) can be written in the form of conservation law with

$$
\mathcal{I}_{0}^{t}=D_{t}^{\alpha-1} u, \quad \mathcal{I}_{0}^{x}=a u_{(n-1) x}+\frac{b}{m+1} u^{m+1} .
$$

In Ref. [16], a new conservation theorem is presented to derive conservation laws for differential equations. According to the new conservation theorem [16], the form Lagrangian for Eq.(1.1) is given by

$$
\mathcal{H}=\psi(x, t)\left(D_{t}^{\alpha} u+a u_{n x}+b u^{m} u_{x}\right)
$$

where $\psi(x, t)$ is a new dependent variable. The adjoint equation of Eq.(1.1) are determined by

$$
\mathcal{F}=\frac{\delta \mathcal{H}}{\delta u}=0,
$$

in which $\frac{\delta}{\delta u}$ is defined by

$$
\frac{\delta}{\delta u}=\frac{\partial}{\partial u}+\left(D_{t}^{\alpha}\right)^{*} \frac{\partial}{\partial D_{t}^{\alpha} u}-D_{x} \frac{\partial}{\partial u_{x}}+D_{x}^{2} \frac{\partial}{\partial u_{x x}}+\sum_{k=3}^{\infty}(-1)^{k} D_{i_{1}} \cdots D_{i_{k}} \frac{\partial}{\partial u_{i_{1}, \cdots, i_{k}}},
$$


and $\left(D_{t}^{\alpha}\right)^{*}$ is the adjoint operator for the $D_{t}^{\alpha}$. In view of the Riemann-Liouville fractional differential operators, we have

$$
\begin{aligned}
& \left(D_{t}^{\alpha}\right)^{*}=(-1)^{n} \mathcal{P}_{T}^{n-\alpha}\left(D_{t}^{n}\right)=\left(D_{T}^{\alpha}\right)_{t}^{\mathcal{I}}, \\
& \mathcal{P}_{T}^{n-\alpha} f(t, x)=\frac{1}{\Gamma(n-\alpha)} \int_{t}^{T} \frac{f(\tau, x)}{(\tau-t)^{1+\alpha-n}} d \tau, \quad n=[\alpha]+1,
\end{aligned}
$$

in which $\left(D_{T}^{\alpha}\right)_{t}^{\mathcal{I}}$ is the right-sided Caputo operator.

Substitution of (5.3) into (5.4) leads to the adjoint equation of Eq.(1.1) admitting the following expression

$$
\mathcal{F}=\left(D_{t}^{\alpha}\right)^{*} \psi+(-1)^{n} \psi_{n x}-b u^{m} \psi=0 .
$$

Invoking Ref. [16], Eq.(1.1) arrives at the following conservation law

$$
D_{t} \mathcal{I}_{i}^{t}+D_{x} \mathcal{I}_{i}^{x}=0
$$

where the conserved vector $\mathcal{I}=\left(\mathcal{I}^{t}, \mathcal{I}^{x}\right)$ has a new form

$$
\begin{aligned}
\mathcal{I}_{i}^{x} & =W_{i} \frac{\delta \mathcal{H}}{\delta u_{x}}+D_{x}\left(W_{i}\right) \frac{\delta \mathcal{H}}{\delta u_{x x}}+\ldots+D_{x}^{n-1}\left(W_{i}\right) \frac{\delta \mathcal{H}}{\delta u_{n x}}, \\
\mathcal{I}_{i}^{t} & =\sum_{k=0}^{n-1}(-1)^{k} D_{t}^{\alpha-1-k}\left(W_{i}\right) D_{t}^{k}\left[\frac{\partial \mathcal{H}}{\partial\left(D_{t}^{\alpha} u\right)}\right]-(-1)^{n} \mathcal{J}\left[W_{i}, D_{t}^{n}\left(\frac{\partial \mathcal{H}}{\partial\left(D_{t}^{\alpha} u\right)}\right)\right], n=[\alpha]+1,
\end{aligned}
$$

here $W_{i}=\eta_{i}-\xi_{i} u_{x}-\tau_{i} u_{t}$ and the integral $\mathcal{J}$ is given by

$$
\mathcal{J}=\frac{1}{\Gamma(n-\alpha)} \int_{0}^{t} \int_{t}^{T} \frac{f(\lambda, x) g(\mu, x)}{(\mu-\lambda)^{\alpha+1-n}} d \lambda d \mu .
$$

Then according to the Lie point symmetries (3.4), we obtain the following conserved vectors for Eqs.(1.1).

Case 1: To the generator $X_{1}=\frac{\partial}{\partial x}$, we obtain the corresponding Lie characteristic function

$$
W_{1}=-u_{x} .
$$

Substitution of (5.11) into (5.9) yields the following conserved vector

$$
\begin{aligned}
\mathcal{I}_{1}^{x} & =W_{1}\left[\frac{\partial \mathcal{H}}{\partial u_{x}}+(-1)^{n} D_{x}^{n-1} \frac{\partial \mathcal{H}}{\partial u_{n x}}\right]+D_{x}^{n-1}\left(W_{1}\right) \frac{\partial \mathcal{H}}{\partial u_{n x}}, \\
\mathcal{I}_{1}^{t} & =-\mathcal{P}_{t}^{1-\alpha}\left(-W_{1}\right) \phi-\mathcal{J}\left(-W_{1}, \phi_{t}\right) .
\end{aligned}
$$

Case 2: To the generator $X_{2}=x \frac{\partial}{\partial x}+\frac{n}{\alpha} t \frac{\partial}{\partial t}+\frac{(1-n)}{p} u \frac{\partial}{\partial u}$, we have the corresponding Lie characteristic function

$$
W_{2}=-x u_{x}-\frac{n t}{\alpha} u_{t}+\frac{(1-n)}{p} u
$$

Substitution of (5.11) into (5.9) yields the following conserved vector

$$
\begin{aligned}
\mathcal{I}_{1}^{x} & =W_{2}\left[\frac{\partial \mathcal{H}}{\partial u_{x}}+(-1)^{n} D_{x}^{n-1} \frac{\partial \mathcal{H}}{\partial u_{n x}}\right]+D_{x}^{n-1}\left(W_{2}\right) \frac{\partial \mathcal{H}}{\partial u_{n x}}, \\
\mathcal{I}_{2}^{t} & =\mathcal{P}_{t}^{1-\alpha}\left(-W_{2}\right) \phi+\mathcal{J}\left(-W_{2}, \phi_{t}\right) .
\end{aligned}
$$


Summing up the above detailed analysis, the following theorem is easily established.

Theorem 5.1 The time-fractional generalized KdV-type equation (1.1) has the following conservation laws

$$
D_{t}\left(\mathcal{I}_{i}^{t}\right)+D_{x}\left(\mathcal{I}_{i}^{x}\right)=0, \quad i=1,2,
$$

where $\mathcal{I}_{i}^{t}$ are shown in (5.2),(5.12) and (5.14).

\section{Conclusions and discussions}

In this work, the time-fractional generalized KdV-type equation (1.1) is systematically investigated, which can be reduced to the ones of several important equations such as KdV (1.2), FS-KdV (1.3), F-Burgers (1.4), FF-KdV (1.5) and so on. Then, all the results obtained here can also be reduced to ones of such important equations. The symmetry properties, similarity reduction forms of (1.1) are constructed by using Lie symmetry method. Besides, based on the sub-equation method, we present the travelling wave solutions for the special forms of Eq.(1.1). Then power series solution of Eq. (1.1) is also constructed by using the power series method. Finally, based on a new conservation theorem [16], conservation laws of Eq.(1.1) are derived with the use of its vector fields (3.4). The paper shows that Lie symmetry analysis method, the sub-equation method and the power series method provide the direct and powerful mathematical tools to further study other fractional differential equations in mathematical physics.

\section{Acknowledgments}

We express our sincere thanks to the Editor and Reviewers for their valuable comments. This work was supported by the Fundamental Research Funds for the Central Universities under Grant No. 2014XT02.

\section{References}

[1] M.J. Ablowitz, P.A. Clarkson, Solitons; Nonlinear Evolution Equations and Inverse Scattering, Cambridge University Press, Cambridge, 1991.

[2] N.H. Asmar, Partial Differential Equations with Fourier Series and Boundary Value Problems. second ed. China Machine Press. Beijing, 2005.

[3] G. W. Bluman, S. Kumei, Symmetries and differential equations.New York. Springer, 1989.

[4] A. Bekir, E. Aksoy and C.A. Cevikel, Exact solutions of nonlinear time-fractional partial differential equations by sub-equation method, Math. Meth. Appl. Sci. 38 (2015) 2779-2784.

[5] Y. Chen, H.L. An, Numerical solutions of coupled Burgers equations with time-and space-fractional derivatives, Appl. Math. Comput. 200 (2008) 87-95.

[6] L. L. Feng, S. F. Tian, X. B. Wang, T. T. Zhang, Lie symmetry analysis, conservation laws and exact power series solutions for time-fractional Fordy-Gibbons equation, Commun. Theor. Phys. 66 (2016) 321.

[7] L. L. Feng, S. F. Tian, X. B. Wang, T. T. Zhang, Rogue waves, homoclinic breather waves and soliton waves for the (2+1)-dimensional B-type Kadomtsev-Petviashvili equation, Appl. Math. Lett. 65 (2017) 90-97.

[8] R.K. Gazizov, A.A. Kasatkin, S.Y. Lukashchuk, Continuous transformation groups of fractional differential equations, Vestn USATU. 9 (2007) 125-135.

[9] R.K. Gazizov, A.A. Kasatkin, S.Y. Lukashchuk, Symmetry properties of fractional diffusion equations, Phys. Scr. (2009) 014-016. 
[10] R.K. Gazizov, N.H. Ibragimov, S.Y. Lukashchuk, Nonlinear self-adjointness, conservation laws and exact solutions of time-fractional Kompaneets equations, Commun. Nonlin. Sci. Numer. Simul. 23 (2015) 153-163.

[11] S. Guo, L. Mei, Y. He, Y. Li, Time-fractional Schamel-KdV equation for dust-ion-acoustic waves in pair-ion plasma with trapped electrons and opposite polarity dust grains, Phys. Lett. A 380 (2016) 1031-1036.

[12] V.A. Galaktionov, S.R. Svirshchevskii, Exact Solutions and Invariant Subspaces of Nonlinear Partial Differential Equations in Mechanics and Physics. Chapman and Hall/CRC, 2006.

[13] Q. Huang, R. Zhdanov, Symmetries and exact solutions of the time-fractional Harry-Dym equation with Riemann-Liouville derivative, Physica A 409 (2014) 110-118.

[14] J. Hu, Y. Ye, S. Shen, J. Zhang, Lie symmetry analysis of the time-fractional KdV-type equation, Appl. Math. Comput. 233 (2014) 439-444.

[15] N. H. Ibragimov, Lie group analysis of differential equations-symmetry, exact solutions and conservation laws. Florida: CRC 2006.

[16] N.H. Ibragimov, A new conservation theorem, J. Math. Anal. Appl. 333 (2007) 311-328.

[17] H. Jafari, G.V. Daftardar, Solving linear and nonlinear fractional diffusion and wave equations by Adomiandecomposition. Appl. Math. Comput. 180 (2006) 488-497.

[18] G. Jumarie, Modified Riemann-Liouville derivative and fractional Taylor series of nondifferentiable functions further results, Comput. Math. Appl. 51 (2006) 1367-1376.

[19] V. S. Kiryakova, Generalised fractional calculus and applications, Longman Scientific Tec, 1994.

[20] S.Y. Lukashchuk, A.V. Makunin, Group classification of nonlinear time-fractional diffusion equation with a source term, Appl Math Comput. 257 (2015) 335-343.

[21] S.Y. Lukashchuk, Conservation laws for time-fractional subdiffusion and diffusion-wave equations, Nonlinear Dyn. 80 (2015) 791-802.

[22] K.S. Miller, B. Ross, An introduction to the fractional calculus and fractional differential equations, New York. Wiley, 1993.

[23] E. Noether, Invariant variational problems, Transp. Theory Stat. Phys. 1 (1971) 186-207.

[24] L. V. Ovsiannikov, Group analysis of differential equations. New York. Academic Press, 1982.

[25] P. J. Over, Application of Lie group to differntial. New York. Springer, 1986.

[26] K.B. Oldham, J. Spanier, The Fractional Calculus. Academic Press, San Diego 1974.

[27] I. Podlubny, Fractional Differential Equations. Academic Press, San Diego 1999.

[28] R. Sahadevan, T. Bakkyaraj, Invariant analysis of time-fractional generalized Burgers and Korteweg-de Vries equations, J. Math. Anal. Appl. 393 (2012) 341-347.

[29] S.F. Tian, H.Q. Zhang, Riemann theta functions periodic wave solutions and rational characteristics for the nonlinear equations, J. Math. Anal. Appl. 371 (2010) 585-608.

[30] J. M. Tu, S.F. Tian, M. J. Xu, X. Q. Song, T. T. Zhang, Bäcklund transformation, infinite conservation laws and periodic wave solutions of a generalized (3+1)-dimensional nonlinear wave in liquid with gas bubbles, Nonlinear Dyn. 83 (2016) 1199-1215.

[31] S. F. Tian, T. T. Zhang, P. L. Ma, X. Y. Zhang, Lie symmetries and nonlocally related systems of the continuous and discrete dispersive long waves system by geometric approach, J. Nonlinear Math. Phys., 22(2) (2015) 180-193.

[32] J. M. Tu, S.F. Tian, M. J. Xu, T. T. Zhang, On Lie symmetries, optimal systems and explicit solutions to the Kudryashov-Sinelshchikov equation, Appl. Math. Comput. 275 (2016) 345-352.

[33] S.F. Tian, H.Q. Zhang, Riemann theta functions periodic wave solutions and rational characteristics for the (1+1)-dimensional and (2+1)-dimensional Ito equation, Chaos, Solitons $\mathcal{E}$ Fractals 47 (2013) 27-41.

[34] S.F. Tian, H.Q. Zhang, On the integrability of a generalized variable-coefficient forced Korteweg-de Vries equation in fluids, Stud. Appl. Math. 132 (2014) 212-246.

[35] J. M. Tu, S.F. Tian, M. J. Xu, T. T. Zhang, Quasi-periodic waves and solitary waves to a generalized KdV-Caudrey-Dodd-Gibbon equation from fluid dynamics, Taiwanese J. Math. 20 (2016) 823-848.

[36] S. F. Tian, Initial-boundary value problems for the general coupled nonlinear Schrödinger equation on the interval via the Fokas method, J. Differ. Equa., 262 (2017) 506-558. 
[37] J. M. Tu, S.F. Tian, M. J. Xu, P. L. Mam T. T. Zhang, On periodic wave solutions with asymptotic behaviors to a (3+1)-dimensional generalized B-type Kadomtsev-Petviashvili equation in fluid dynamics, Comput. Math. Appl. 72 (2016) 2486-2504.

[38] S. F. Tian, The mixed coupled nonlinear Schrödinger equation on the half-line via the Fokas method, Proc. R. Soc. Lond. A 472 (2016) 20160588.

[39] G.W. Wang, T.Z. Xu, Symmetry properties and explicit solutions of the nonlinear time-fractional KdV equation, Boundary Value Prob. 2013 (2013) 232(13pp).

[40] L. Wang, S. F. Tian, Z. T. Zhao, X. Q. Song, Lie symmetry analysis and conservation laws of a generalized time fractional Foam Drainage equation, Commun. Theor. Phys. 66 (2016) 35.

[41] X.B. Wang, S.F. Tian, C.Y. Qin and T.T. Zhang, Lie symmetry analysis, conservation laws and exact solutions of the generalized time-fractional Burgers equation, EPL 114 (2016) 20003.

[42] G.W. Wang, X.Q. Liu, Y.Y. Zhang, Lie symmetry analysis to the time-fractional generalized fifth-order KdV equation, Commun. Nonlinear Sci. Numer. Simul. 18 (2013) 2321-2326.

[43] X. B. Wang, S. F. Tian, C. Y. Qin, T. T. Zhang, Dynamics of the breathers, rogue waves and solitary waves in the (2+1)-dimensional Ito equation, Appl. Math. Lett. 68 (2017) 40-47.

[44] X. B. Wang, S. F. Tian, C. Y. Qin, T. T. Zhang, Characteristics of the solitary waves and rogue waves with interaction phenomena in a generalized $(3+1)$-dimensional Kadomtsev-Petviashvili equation, Appl. Math. Lett. 72 (2017) 58-64.

[45] X. B. Wang, S. F. Tian, M. J. Xu, T. T. Zhang, On integrability and quasi-periodic wave solutions to a (3+1)-dimensional generalized KdV-like model equation, Appl. Math. Comput. 283 (2016) 216-233.

[46] X. B. Wang, S. F. Tian, L. L. Feng, H. Yan, T. T. Zhang, Quasiperiodic waves, solitary waves and asymptotic properties for a generalized $(3+1)$-dimensional variable-coefficient B-type KadomtsevPetviashvili equation, Nonlinear Dyn. 88 (2017) 2265-2279.

[47] M. J. Xu, S. F. Tian, J. M. Tu, T. T. Zhang, Bäcklund transformation, infinite conservation laws and periodic wave solutions to a generalized (2+1)-dimensional Boussinesq equation, Nonlinear Anal.: RWA 31 (2016) 388-408.

[48] S. Zhang, H.Q. Zhang, Fractional sub-equation method and its applications to nonlinear fractional PDEs, Phys. Lett. A 375 (2011) 1069-1073. 